\section{Influence of female sex and fertile age on neuromyelitis optica spectrum disorders}

\author{
Nadja Borisow, Ingo Kleiter, Anna Gahlen, Katrin Fischer, Klaus-Dieter Wernecke, \\ Florence Pache, Klemens Ruprecht, Joachim Havla, Markus Krumbholz, \\ Tania Kümpfel, Orhan Aktas, Marius Ringelstein, Christian Geis, Christoph Kleinschnitz, \\ Achim Berthele, Bernhard Hemmer, Klemens Angstwurm, Robert Weissert, \\ Jan-Patrick Stellmann, Simon Schuster, Martin Stangel, Florian Lauda, \\ Hayrettin Tumani, Christoph Mayer, Lena Zeltner, Ulf Ziemann, Ralf A Linker, \\ Matthias Schwab, Martin Marziniak, Florian Then Bergh, Ulrich Hofstadt-van Oy, \\ Oliver Neuhaus, Alexander Winkelmann, Wael Marouf, Lioba Rückriem, Jürgen Faiss, \\ Brigitte Wildemann, Friedemann Paul, Sven Jarius, Corinna Trebst and Kerstin Hellwig; on \\ behalf of NEMOS (Neuromyelitis Optica Study Group)
}

\section{Abstract}

Background: Gender and age at onset are important epidemiological factors influencing prevalence, clinical presentation, and treatment response in autoimmune diseases.

Objective: To evaluate the impact of female sex and fertile age on aquaporin-4-antibody (AQP4-ab) status, attack localization, and response to attack treatment in patients with neuromyelitis optica (NMO) and its spectrum disorders (neuromyelitis optica spectrum disorder (NMOSD)).

Methods: Female-to-male ratios, diagnosis at last visit (NMO vs NMOSD), attack localization, attack treatment, and outcome were compared according to sex and age at disease or attack onset.

Results: A total of $186 \mathrm{NMO} / \mathrm{SD}$ patients ( $82 \%$ female) were included. In AQP4-ab-positive patients, female predominance was most pronounced during fertile age (female-to-male ratio 23:1). Female patients were more likely to be positive for AQP4-abs $(92 \%$ vs $55 \% ; p<0.001)$. Interval between onset and diagnosis of NMO/SD was longer in women than in men (mean 54 vs 27 months; $p=0.023$ ). In women, attacks occurring $\leqslant 40$ years of age were more likely to show complete remission $(p=0.003)$ and better response to high-dose intravenous steroids $(p=0.005)$ compared to woman at $>40$ years.

Conclusion: Our data suggest an influence of sex and age on susceptibility to AQP4-ab-positive NMO/ SD. Genetic and hormonal factors might contribute to pathophysiology of NMO/SD.

Keywords: Neuromyelitis optica, sex, age factors, aquaporin 4

Date received: 20 April 2016; revised: 15 July 2016; accepted: 4 August 2016

\section{Introduction}

Neuromyelitis optica (NMO) and its spectrum disorders (neuromyelitis optica spectrum disorder (NMOSD)) are autoimmune disorders of the central nervous system, diagnostically characterized and pathophysiologically linked with the presence of antiaquaporin-4-antibodies (AQP4-abs) in the majority of patients. ${ }^{1}$ The female-to-male ratio markedly exceeds $3: 1^{2,3}$ and reaches $8-9: 1$ in some AQP4-ab-positive populations. ${ }^{4}$ With clear female predominance in most studies, sex emerges as one of the most important factors of susceptibility to NMO/SD.
Sex may also influence clinical features, disease course, and severity in NMO/SD. Previous studies showed a female predominance in AQP4-abseropositive patients. ${ }^{4,5}$ Male NMO patients were more likely to follow a monophasic disease course in a predominantly Caucasian cohort. ${ }^{6}$

Over the lifespan, women undergo tremendous hormonal changes, especially during puberty, pregnancy, and menopause. These hormonal changes might influence susceptibility, course, and severity of autoimmune diseases. Other autoimmune disorders, for
Multiple Sclerosis Journal

2017, Vol. 23(8) 1092-1103

DOI: $10.1177 /$

1352458516671203

(C) The Author(s), 2016. Reprints and permissions: http://www.sagepub.co.uk/ journalsPermissions.nav

Correspondence to:

N Borisow

NeuroCure Clinical Research

Center and Clinical and

Experimental Multiple

Sclerosis Research Center,

Department of Neurology,

Charité-Universitätsmedizin

Berlin, Charitéplatz 1, 10117

Berlin, Germany.

nadja.borisow@charite.de

Nadja Borisow

Florence Pache

NeuroCure Clinical Research

Center and Clinical and

Experimental Multiple

Sclerosis Research Center,

Department of Neurology,

Charité University Medicine

Berlin, Berlin, Germany

Friedemann Paul

NeuroCure Clinical Research

Center and Clinical and Experimental Multiple

Sclerosis Research Center,

Department of Neurology,

Charité University Medicine

Berlin, Berlin, Germany/

Experimental and Clinical

Research Center, Max

Delbrueck Center for

Molecular Medicine and

Charité University Medicine Berlin, Berlin, Germany

Ingo Kleiter

Anna Gahlen

Kerstin Hellwig

Department of Neurology,

St. Josef Hospital, Ruhr

University Bochum, Bochum,

Germany

Katrin Fischer

Jürgen Faiss

Department of Neurology,

Asklepios Fachklinikum

Teupitz, Teupitz, Germany

Klaus-Dieter Wernecke

CRO SOSTANA GmbH and

Charité University Medicine

Berlin, Berlin, Germany

Klemens Ruprecht

Department of Neurology and

Clinical and Experimental

Multiple Sclerosis Research

Center, Charité University

Medicine Berlin, Berlin,

Germany

Joachim Havla

Markus Krumbholz

Tania Kümpfel

Institute of Clinical

Neuroimmunology, Medical

Campus Grosshadern, 
example, systemic lupus erythematosus (SLE) ${ }^{7}$ or multiple sclerosis (MS) ${ }^{8}$ show a clear female predominance. In SLE, female-to-male ratio is most pronounced during fertile age. ${ }^{7}$ In MS, male patients are older, more likely to develop myelopathy, and suffer from faster progression than female patients. ${ }^{9}$

Increase in knowledge on sex- and age-specific aspects might be helpful for prognostic assessment and open insights in the susceptibility and pathophysiology of $\mathrm{NMO} / \mathrm{SD}$.

In this study, we investigated for the first time if there are (1) differences in the female-to-male ratio between age groups in NMO/SD and (2) differences in symptoms of attacks and treatment response in female patients during and beyond reproductive age.

\section{Methods}

Patients were identified using the registry of the German Neuromyelitis Optica Study Group (NEMOS, www.nemos-net.de), a nationwide open association of neurological centers interested mainly in adult NMO/SD (34 German university and academic teaching hospitals). Pediatric centers were not participating in the current study. The study was approved by the institutional review boards of the participating academic centers and conducted in accordance with the German data protection law. Between January 2012 and March 2013, we retrospectively analysed data of $215 \mathrm{NMO} / \mathrm{SD}$ patients which were collected crosssectionally in a standardized manner by two neurologists visiting the contributing centers in a "flying doctor" approach.

Inclusion criterion was a diagnosis of NMO according to Wingerchuk et al.'s criteria ${ }^{10}$ ("NMO") or of isolated or recurrent AQP4-ab-positive optic neuritis $(\mathrm{ON})$ or myelitis ("NMOSD"). ${ }^{11}$ Some patients of our cohort were initially misdiagnosed as MS. To avoid misclassification, the diagnosis at the most recent visit prior to data collection was used for patient stratification. Demographic characteristics, diagnoses, duration of clinical observation, clinical attacks, attack treatment, and attack outcome were analysed in a stratified fashion according to sex and to fertile age in women.

The vast majority of girls experience menarche around the age of 15 years, and only a very small proportion of births occurs before the 15th or after the 40th year of age. ${ }^{12}$ Moreover, menopause is very unlikely to occur before the age of 40 years; the risk of natural menopause before age 40 is approximately
1\%. ${ }^{13}$ Therefore, we defined fertile age between 15 and 40 years. Time to diagnosis, AQP4-ab status, disease classification, and the annualized relapse rate (ARR) were analysed according to age at disease onset. Attack-related factors like clinical presentation and attack remission were at first analysed according to age at attack onset. Attack treatment was escalated up to four times. Only first treatment courses for attacks were included in the analysis of treatment responses.

Of 215 patients recorded in the database, 29 patients were excluded due to missing data or because they did not meet inclusion criteria. Finally, data from 186 patients were available for statistical analysis (Figure 1). These patients had a total of 1124 attacks, but in 253 attacks, documentation on treatment and/ or outcome was insufficient. Therefore, only 871 attacks were included in the final analysis.

Demographic data, attack characteristics, therapies, and the short-term remission status of the complete cohort were previously described. ${ }^{14}$

The statistical analysis was conducted in two steps. First, we analysed patient-related data such as age at onset, time between onset and diagnosis, AQP4-ab serostatus, disease classification, and ARR. Those analyses were accomplished using the exact chisquare test or the exact Mann-Whitney test, accordingly. Second, in order to adjust for intraindividual correlations within patients, we applied generalized estimating equations (GEEs) ${ }^{15}$ with patient as statistical unit for attack localization (type of attack) and attack remission. In GEE, odds ratios for risk factors and corresponding $95 \%$ confidence intervals $(95 \%$ CIs) were given. Statistical analyses were performed using IBM(C) SPSSC Statistics, Version 23, CCopyright 1989, 2010 SPSS Inc., an IBM Company. Results are shown as median and interquartile range (IQR) or mean \pm standard deviation (SD) when normal distribution wasn't rejected. Statistical significance was set at a two-sided $p<0.05$. Our study was an exploratory analysis; therefore, no correction for multiple comparisons was performed.

\section{Results}

\section{Female-to-male ratio in different age groups}

We identified a total of 186 patients (Figure 1). Age at disease onset ranged between 15 and 65 years in more than $90 \%$ of the patients. The youngest patient was 8 years old at disease onset, whereas the oldest developed first symptoms at an age of 79 years (Table 1). In
Ludwig Maximilians University of Munich, Munich, Germany

Orhan Aktas

Marius Ringelstein

Department of Neurology, Medical Faculty, Heinrich

Heine University Düsseldorf, Düsseldorf, Germany

Christoph Kleinschnitz Department of Neurology, University Hospital Essen, Essen, Germany

Christian Geis

Department of Neurology,

University Hospital of

Würzburg, Würzburg, Germany/Hans-Berger

Department of Neurology and Center for Sepsis Control and Care (CSCC), Jena University Hospital, Jena, Germany

Achim Berthele

Department of Neurology,

Klinikum rechts der Isar,

Technische Universität

München, Munich, Germany

Bernhard Hemmer

Department of Neurology,

Klinikum rechts der Isar,

Technische Universität

München, Munich, Germany/

Munich Cluster for Systems

Neurology (SyNergy),

Technische Universität

München, Munich, Germany

Klemens Angstwurm

Robert Weissert

Department of Neurology,

University Hospital

Regensburg, Regensburg,

Germany

Jan-Patrick Stellmann

Institute of

Neuroimmunology and MS

(INIMS), University Medical

Center Hamburg-Eppendorf,

Hamburg, Germany/

Department of Neurology,

University Medical Center

Hamburg-Eppendorf,

Hamburg, Germany

Simon Schuster

Department of Neurology,

University Medical Center

Hamburg-Eppendorf,

Hamburg, Germany

Martin Stangel

Department of Clinical

Neuroimmunology and

Neurochemistry and

Department of Neurology,

Hannover Medical School,

Hannover, Germany

Florian Lauda

Department of Neurology,

University of Ulm, Ulm,

Germany

Hayrettin Tumani

Department of Neurology at

RKU and Specialty Clinic

of Neurology Dietenbronn,

University of Ulm, Ulm,

Germany

Christoph Mayer

Department of Neurology,

Goethe University Frankfurt,

Frankfurt, Germany 
Lena Zeltner

Ulf Ziemann

Department of Neurology and

Stroke and Hertie Institute

for Clinical Brain Research,

University of Tübingen,

Tübingen, Germany

Ralf A Linker

Department of Neurology,

Friedrich-Alexander

University Erlangen-

Nüremberg, Erlangen,

Germany

Matthias Schwab

Hans-Berger Department of

Neurology, Jena University

Hospital, Jena, Germany

Martin Marziniak

Department of Neurology,

University of Münster,

Münster, Germany/

Department of Neurology

and Neurological Intensive

Care, Isar-Amper-Clinic,

Munich-East, Haar, Germany

Florian Then Bergh

Department of Neurology,

Leipzig University, Leipzig,

Germany

Ulrich Hofstadt-van Oy

Department of Neurology,

Klinikum Bayreuth,

Bayreuth, Germany/

Department of Neurology,

Klinikum Westfalen,

Dortmund, Germany

Oliver Neuhaus

Department of Neurology,

SRH Krankenhaus

Sigmaringen, Sigmaringen,

Germany

Alexander Winkelmann

Department of Neurology,

University of Rostock,

Rostock, Germany

Wael Marouf

Department of Neurology,

HELIOS Hanseklinikum

Stralsund, Stralsund,

Germany

Lioba Rückriem

Department of Neurology,

MediClin Hedon Klinik,

Lingen, Germany

Brigitte Wildemann

Sven Jarius

Department of Neurology,

Heidelberg University,

Heidelberg, Germany

Corinna Trebst

Department of Neurology,

Hannover Medical School,

Hannover, Germany

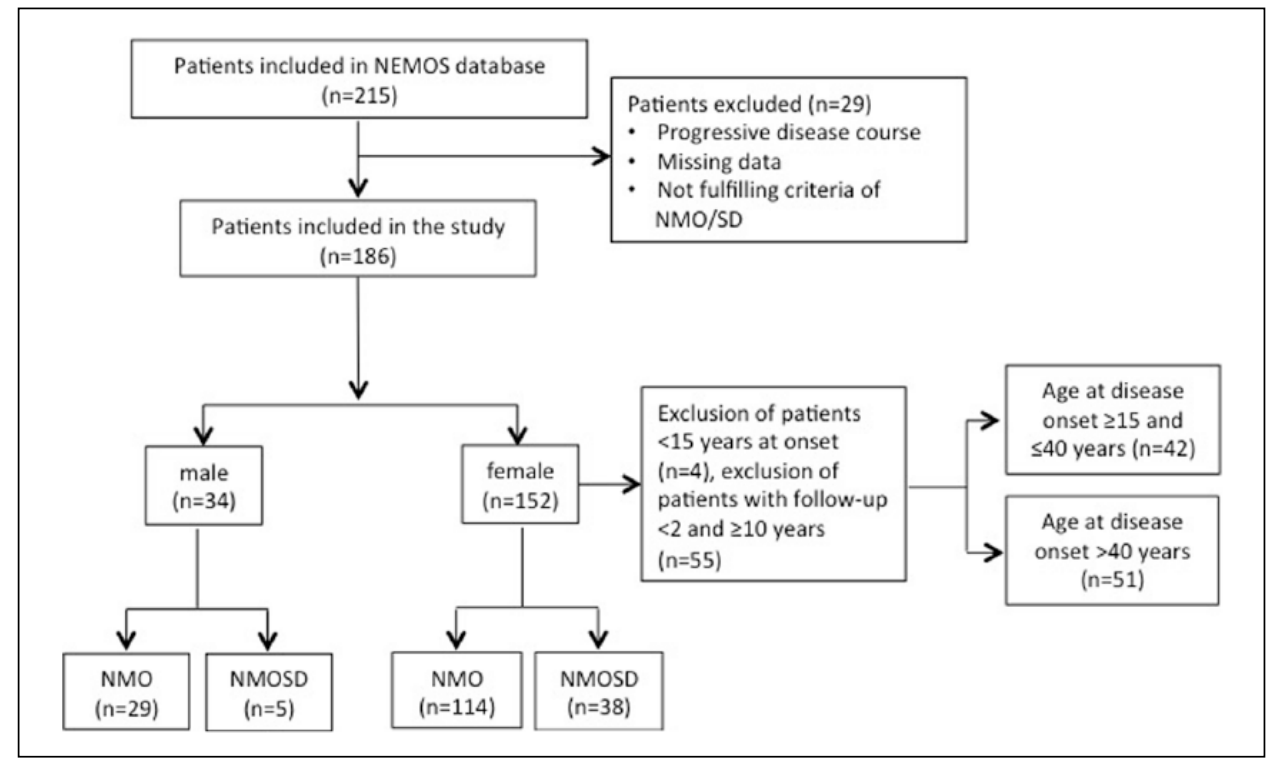

Figure 1. Flow chart of the patients enrolled in the study.

Table 1. Demographic characteristics.

\begin{tabular}{llc}
\hline & & $n(\%)$ \\
\hline Sex & Female & $152 / 186(82)$ \\
& Male & $34 / 186(18)$ \\
Age at onset & $<15$ years & $6 / 186(3)$ \\
(complete cohort, & $15-40$ years & $89 / 186(48)$ \\
range: 8-79) & $41-65$ years & $83 / 186(45)$ \\
& $>65$ years & $8 / 186(4)$ \\
Age at onset & $<15$ years & $4 / 152(3)$ \\
(women only) & $15-40$ years & $78 / 152(51)$ \\
& $41-65$ years & $65 / 152(43)$ \\
& $>65$ years & $5 / 152(3)$ \\
Age at onset & $<15$ years & $2 / 34(6)$ \\
(men only) & $15-40$ years & $10 / 34(29)$ \\
& $41-65$ years & $19 / 34(56)$ \\
& $>65$ years & $3 / 34(9)$ \\
\hline
\end{tabular}

all, $152(82 \%)$ were female. Female-to-male ratio was $4.5: 1$ in the total cohort, $8: 1$ in patients with disease onset in the fertile age, and 3:1 in patients older than 40 years at disease onset (Figure 2). In the small subgroup of patients $<15$ years at onset $(n=6)$, female-tomale ratio was $2: 1$. In $183 / 186$ patients (98\%), information about AQP4-ab-status were available. Out of these, 156 (85\%) were AQP4-ab-positive. In AQP4-ab-positive patients, female-to-male ratios were $3: 1,23: 1$, and 5:1 for age groups $<15,15-40$, and $>40$ years, respectively. In contrast, in AQP4-abnegative patients $(n=27,15 \%)$, the female-to-male ratios were $1: 1.2$ for age groups $15-40$ and $>40$ years, respectively. As in AQP4-ab-negative patients only one patient was younger than 15 years at onset, a female-to-male ratio was not applicable.

\section{Comparison between female and male patients}

Mean duration of clinical observation in female and male patients was $47(23-107)$ versus 68 (39$117)$ months $(p=0.054)$. Women tended to be younger (mean $39 \pm 14$ years) than men (mean $44 \pm 17$ years, $p=0.075)$ at disease onset. The majority of both men and women showed a relapsing disease course $(91 \%$ and $94 \%$, respectively), while in the remaining patients, a monophasic disease course was recorded. In women, the duration until the diagnosis of $\mathrm{NMO} /$ SD had been confirmed was longer than in men $(p=0.023)$. AQP4-abs were more frequently detected in female than in male patients $(p<0.001$; Table 2$)$.

The number of female and male patients receiving disease-modifying treatments (DMTs) is shown in Table 2 . The median number of DMT per patient was 2 (range 1-8). Mean DMT interval was 441 ( \pm 421$)$ days.

A total of $28 \%$ of all attacks presented as ON and $59 \%$ as myelitis. The remaining attacks showed either simultaneous $\mathrm{ON}$ and myelitis or symptoms different from $\mathrm{ON}$ or myelitis. No differences in the frequencies of $\mathrm{ON}$ and myelitis attacks were found between male and female patients (Table 2).

In almost all patients $(181 / 186,97 \%)$, at least once an acute attack was treated with high-dose intravenous 


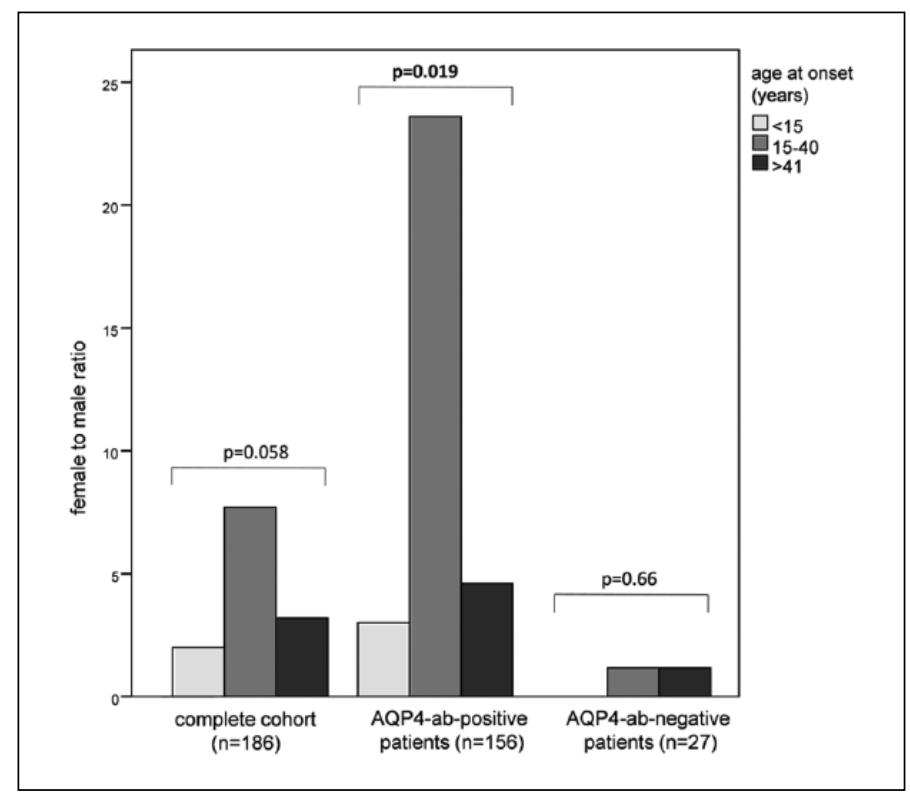

Figure 2. Age distribution of female-to-male ratio.

steroids (HD-S) as first-line treatment. A total of 27 patients $(15 \%)$ received therapeutic plasma exchange (TPE) and 7 (4\%) received immunoadsorption as first treatment course of at least one attack. Frequencies of applied first-line therapies did not differ between men and women. No differences in attack localization and in attack outcome for any of the applied first treatment courses were found between male and female patients (Table 2).

\section{Comparison between women of fertile age and women beyond fertile age}

Female patients younger than 15 years $(n=4)$ were not included in the analysis. To ensure a similar clinical observation period in both groups, female patients with an observation period of $<2$ and $\geqslant 10$ years $(n=55)$ were excluded from the analysis. Among the remaining 93 women, 42 were between 15 and 40 years old at disease onset (median clinical observation 74 (40-88) months). A total of 51 female patients were older than 40 years (median clinical observation $51(40-72)$ months; $p=0.08)$ at disease onset. A total of 468 attacks were recorded in these patients.

Women $\leqslant 40$ years at disease onset were more likely to fulfill Wingerchuk's criteria compared to women $>40$ years $(p=0.008)$. No differences were detected in AQP4-ab status, ARR, and time between initial manifestation and diagnosis (Table 3 ).
We found no differences in the use of first-line attack therapy between women $\leqslant 40$ years at disease onset and female patients $>40$ years.

Attacks in women occurring at an age of 40 years or lower showed a higher frequency of complete attack remission $(p=0.003)$ and a better response to HD-S $(p=0.005)$. The comparison of response to TPE between attacks occurring at an age of $\leqslant$ and $>40$ years missed significance. No differences in attack localization were detected (Table 3).

In order to differentiate between age and sex effects, we also explored the male patients. Male patients between 15 and 40 years at disease onset $(n=10)$ did not differ from male patients $>40$ years $(n=22)$ with regard to ARR and disease classification (NMO vs NMOSD). No differences in attack localization, attack outcome, or treatment response to HD-S and TPE were detected in attacks occurring at an age of 40 years or lower compared to attacks occurring at an age of $>40$ years (Table 4 ).

\section{Discussion}

In our cohort of predominantly Caucasian NMO/SD patients, we found a significant female preponderance. A total of $82 \%$ of our patients were female, which is in line with the results of other NMO cohorts. ${ }^{3}$ The female-to-male ratio at disease onset was most pronounced in the reproductive age between 15 and 40 years (8:1 vs 2:1 and 3:1, respectively). 
Table 2. Comparison between female $(n=152)$ and male $(n=34) \mathrm{NMO} / \mathrm{SD}$ patients.

\begin{tabular}{|c|c|c|c|c|}
\hline & $\begin{array}{l}\text { Female }(n=152) \\
\text { (reference) }\end{array}$ & Male $(n=34)$ & $p$-value & Odds ratio $(95 \% \mathrm{CI})$ \\
\hline Age at onset (years $( \pm \mathrm{SD}))$ & $39( \pm 14)$ & $44( \pm 17)$ & $0.08^{\mathrm{a}}$ & \\
\hline $\begin{array}{l}\text { Time between onset and } \\
\text { diagnosis (months) }\end{array}$ & $54( \pm 80)$ & $27( \pm 42)$ & $0.02^{\mathrm{a}}$ & \\
\hline AQP4-ab-positive & $138(92 \%)$ & $18(55 \%)$ & $<0.001^{\text {b }}$ & \\
\hline $\begin{array}{l}\text { Annualized relapse rate } \\
\text { (clinical observation }>1 \text { year) }\end{array}$ & $0.9(0.6-1.4)$ & $0.9(0.6-1.4)$ & $0.90^{\mathrm{a}}$ & \\
\hline \multicolumn{5}{|l|}{ Disease classification } \\
\hline $\mathrm{NMO}^{\mathrm{c}}$ & $114 / 152(75 \%)$ & $29 / 34(85 \%)$ & $0.20^{\mathrm{b}}$ & \\
\hline NMOSD & $38 / 152(25 \%)$ & $5 / 34(15 \%)$ & & \\
\hline \multicolumn{5}{|c|}{ Disease-modifying treatments (patients receiving treatment $n$ ) } \\
\hline Rituximab & $62-a$ & 12 & & \\
\hline Azathioprine & 50 & 4 & & \\
\hline Mitoxantrone & 29 & 9 & & \\
\hline Interferon-beta & 26 & 5 & & \\
\hline Glatiramer acetate & 17 & 1 & & \\
\hline Cyclophosphamide & 16 & 0 & & \\
\hline Steroids per os & 14 & 2 & & \\
\hline Steroids intrathecal & 7 & 2 & & \\
\hline Others $^{\mathrm{d}}$ & 29 & 8 & & \\
\hline \multicolumn{5}{|c|}{ Type of attack ( $n=871$ attacks in 185 patients) } \\
\hline Optic neuritis $(\mathrm{ON})$ & 213/731 (29) & $35 / 140(25)$ & $0.69^{\mathrm{e}}$ & $1.049(0.825-1.34)$ \\
\hline Myelitis & $425 / 731(58)$ & $92 / 140(66)$ & & \\
\hline $\mathrm{ON}+$ myelitis & $77 / 731(11)$ & $12 / 140(8)$ & & \\
\hline Other & $16 / 731(2)$ & $1 / 140(1)$ & & \\
\hline \multicolumn{5}{|c|}{ Remission overall ( $n=871$ attacks in 185 patients) } \\
\hline Complete & $147 / 731(20)$ & $26 / 140(19)$ & $0.69 \mathrm{e}$ & $0.888(0.496-1.59)$ \\
\hline Incomplete/no & $573 / 731(78)$ & $112 / 140(80)$ & & \\
\hline MD & $11 / 731(2)$ & $2 / 140(1)$ & & \\
\hline \multicolumn{5}{|c|}{ Remission HD-S (first pulse) ( $n=693$ attacks in 181 patients) } \\
\hline Complete & $96 / 575(17)$ & 22/118 (19) & $0.76^{\mathrm{e}}$ & $1.102(0.591-2.06)$ \\
\hline Incomplete/no & $471 / 575(82)$ & $94 / 118(80)$ & & \\
\hline MD & $8 / 575(1)$ & $2 / 118(1)$ & & \\
\hline \multicolumn{5}{|c|}{ Remission TPE (first cycle) ( $n=63$ attacks in 28 patients) } \\
\hline Complete & $18 / 55(33)$ & $1 / 8(13)$ & $0.76^{\mathrm{e}}$ & $0.704(0.075-6.57)$ \\
\hline Incomplete/no & $36 / 55(65)$ & $7 / 8(87)$ & & \\
\hline MD & $1 / 55(2)$ & $0 / 8(0)$ & & \\
\hline \multicolumn{5}{|c|}{ Remission immunoadsorption (first cycle) ( $n=9$ attacks in seven patients) } \\
\hline Complete & $0 / 8(0)$ & $1 / 1(100)$ & NA & \\
\hline Incomplete/no & $8 / 8(100)$ & $0 / 1(0)$ & & \\
\hline
\end{tabular}

HD-S: high-dose intravenous steroids; TPE: therapeutic plasma exchange; NA: not applicable; MD: missing data; CI: confidence interval; SD: standard deviation; NMO: neuromyelitis optica; NMOSD: neuromyelitis optica spectrum disorder; AQP4-ab: aquaporin-4 antibody.

aMann-Whitney $U$ test was performed to compare continuous data.

${ }^{\mathrm{b}}$ Categorical data were compared using exact chi-square test.

'NMO fulfilling Wingerchuk's criteria. ${ }^{10}$

dAlemtuzumab, cyclosporine A, fingolimod, intravenous immunoglobulins, steroids intravenous, tocilizumab, natalizumab, mycophenolate mofetile, and methotrexate.

${ }^{\mathrm{e}}$ To adjust for intraindividual dependencies we additionally applied generalized estimating equations (GEEs) with patient as statistical unit for analysis. Shown are median and interquartile range (IQR) or mean $\pm \mathrm{SD}$ when normally distributed. 
Table 3. Comparison between female NMO/SD patients of fertile age and beyond fertile age at onset.

\begin{tabular}{|c|c|c|c|c|}
\hline & $\begin{array}{l}\text { Age between } 15 \text { and } \\
40 \text { years }(n=42)\end{array}$ & $\begin{array}{l}\text { Age }>40 \text { years } \\
(n=51)(\text { reference })\end{array}$ & $p$-value & Odds ratio $(95 \% \mathrm{CI})$ \\
\hline $\begin{array}{l}\text { Time between onset and } \\
\text { diagnosis (months) }\end{array}$ & $31( \pm 31)$ & $22( \pm 21)$ & $0.25^{\mathrm{a}}$ & \\
\hline AQP4-ab-positive & $38 / 42(91 \%)$ & $49 / 51(96 \%)$ & $0.27^{\mathrm{b}}$ & \\
\hline $\begin{array}{l}\text { Annualized relapse rate (clinical } \\
\text { observation } \geqslant 2 \text { and }<10 \text { years) }\end{array}$ & $1.1( \pm 0.7)$ & $1.0( \pm 0.6)$ & $0.98^{\mathrm{a}}$ & \\
\hline \multicolumn{5}{|l|}{ Disease classification } \\
\hline $\mathrm{NMO}^{\mathrm{c}}$ & $36 / 42(86 \%)$ & $31 / 51(61 \%)$ & \multirow[t]{2}{*}{$\mathbf{0 . 0 0 8}^{\mathrm{b}}$} & \\
\hline NMOSD & $6 / 42(14 \%)$ & $20 / 51(39 \%)$ & & \\
\hline \multicolumn{5}{|c|}{ Type of attack ( $n=468$ attacks in 93 patients) } \\
\hline Optic neuritis $(\mathrm{ON})$ & $69 / 226(31)$ & $58 / 242(24)$ & \multirow[t]{4}{*}{$0.10^{\mathrm{d}}$} & \multirow[t]{4}{*}{$0.803(0.618-1.04)$} \\
\hline Myelitis & $129 / 226(57)$ & $155 / 242(64)$ & & \\
\hline $\mathrm{ON}+$ myelitis & $19 / 226(8)$ & $28 / 242(12)$ & & \\
\hline Others & $9 / / 226(4)$ & $1 / 242(0.4)$ & & \\
\hline \multicolumn{5}{|c|}{ Remission overall ( $n=468$ attacks in 93 patients) } \\
\hline Complete & $57 / 226(25)$ & $29 / 242(12)$ & \multirow[t]{3}{*}{$\mathbf{0 . 0 0 3}^{\mathrm{d}}$} & \multirow[t]{3}{*}{$0.361(0.185-0.705)$} \\
\hline Incomplete/no & $136 / 226(60)$ & $189 / 242(78)$ & & \\
\hline MD & $33 / 226(15)$ & $24 / 242(10)$ & & \\
\hline \multicolumn{5}{|c|}{ Remission HD-S (first pulse) ( $n=338$ attacks in 93 patients) } \\
\hline Complete & $32 / 155(21)$ & $19 / 183(10)$ & \multirow[t]{3}{*}{$0.005^{\mathrm{d}}$} & \multirow[t]{3}{*}{$0.353(0.172-0.728)$} \\
\hline Incomplete/no & $108 / 155(70)$ & $156 / 183(85)$ & & \\
\hline MD & $15 / 155(10)$ & $9 / 183(5)$ & & \\
\hline \multicolumn{5}{|c|}{ Remission TPE (first cycle) ( $n=38$ attacks in 15 patients) } \\
\hline Complete & $15 / 18(83)$ & $0 / 20(0)$ & \multirow[t]{3}{*}{ NA } & \multirow[t]{3}{*}{ No solution for GEE } \\
\hline Incomplete/no & $2 / 18(11)$ & $19 / 20(95)$ & & \\
\hline MD & $1 / 18(6)$ & $1 / 20(5)$ & & \\
\hline \multicolumn{5}{|c|}{ Remission immunoadsorption (first cycle) ( $n=$ seven attacks in five patients) } \\
\hline Complete & $0 / 7(0)$ & $0 / 0(0)$ & \multirow[t]{2}{*}{ NA } & \\
\hline Incomplete/no & $7 / 7(100)$ & $0 / 0(0)$ & & \\
\hline \multicolumn{5}{|c|}{$\begin{array}{l}\text { HD-S: high-dose intravenous steroids; TPE: therapeutic plasma exchange; NA: not applicable; MD: missing data; CI: confidence } \\
\text { interval; SD: standard deviation; NMO: neuromyelitis optica; NMOSD: neuromyelitis optica spectrum disorder; AQP4-ab: aquapo- } \\
\text { rin-4 antibody. } \\
\text { To ensure a similar duration of clinical observation in both groups, only women with an observation period of }>2 \text { and } \leqslant 10 \text { years } \\
\text { were included in the analysis. } \\
\text { ann-Whitney } U \text { test was performed to compare continuous data. } \\
\text { bCategorical data were compared using exact chi-square test. } \\
\text { 'NMO fulfilling Wingerchuk's criteria. } \\
\text { dTo } \\
\text { dTo adjust for intraindividual dependencies, we additionally applied generalized estimating equations (GEEs) with patient as statisti- } \\
\text { cal unit for analysis. Shown are median and interquartile range (IQR) or mean } \pm \text { SD when normally distributed. }\end{array}$} \\
\hline
\end{tabular}

Many autoimmune diseases show a female predominance, for example, SLE and MS. As in our NMO/SD study, female preponderance is also marked during fertile age in SLE. ${ }^{16}$ Studies in pediatric MS showed a balanced sex ratio in children $<11$ years ${ }^{17}$ and highest female-to-male ratios in patients between 12 and 15 years, ${ }^{17,18}$ suggesting that hormonal changes during puberty have an impact on MS onset; female-tomale ratio declined with increasing age and showed a male excess in MS patients with disease onset after the 50 th year of age..$^{18}$
Previous studies described a strong female predominance in pediatric NMO. ${ }^{2,19}$ In our cohort, the number of patients with pediatric onset was very low $(n=6)$. We cannot rule out that the female-to-male ratio of 2:1 found in this age group is due to low patient numbers or to differences in ethnic background between studies.

Diagnosis of NMO/SD was significantly delayed in women compared to men. Being misdiagnosed with MS was the main reason for this delay. Prior to the 
Table 4. Comparison between male NMO/SD patients between 15 and 40 years and $>40$ years of age.

\begin{tabular}{|c|c|c|c|c|}
\hline & $\begin{array}{l}\text { Age between } 15 \text { and } \\
40 \text { years }(n=10)\end{array}$ & $\begin{array}{l}\text { Age }>40 \text { years } \\
(n=22)\end{array}$ & $p$-value & Odds ratio $(95 \% \mathrm{CI})$ \\
\hline $\begin{array}{l}\text { Time between onset and } \\
\text { diagnosis (months) }\end{array}$ & $37( \pm 56)$ & $16( \pm 26)$ & $0.41^{\mathrm{a}}$ & \\
\hline AQP4-ab-positive & $3(30 \%)$ & $14(64 \%)$ & $0.12^{b}$ & \\
\hline $\begin{array}{l}\text { Annualized relapse rate (clinical } \\
\text { observation }>1 \text { year) }\end{array}$ & $1.0(0.7-1.5)$ & $1.0(0.7-1.8)$ & $0.62^{\mathrm{a}}$ & \\
\hline \multicolumn{5}{|l|}{ Disease classification } \\
\hline $\mathrm{NMO}^{\mathrm{c}}$ & $10(100 \%)$ & $18(82 \%)$ & \multirow[t]{2}{*}{$0.15^{\mathrm{b}}$} & \\
\hline NMOSD & $0(0 \%)$ & $4(18 \%)$ & & \\
\hline \multicolumn{5}{|c|}{ Type of attack ( $n=135$ in 33 patients) } \\
\hline Optic neuritis (ON) & $14 / 51$ & $17 / 84$ & \multirow[t]{4}{*}{$0.71^{\mathrm{d}}$} & \multirow[t]{4}{*}{$0.917(0.582-1.45)$} \\
\hline Myelitis & $32 / 51$ & $59 / 84$ & & \\
\hline $\mathrm{ON}+$ myelitis & $5 / 51$ & $7 / 84$ & & \\
\hline Other & $0 / 51$ & $1 / 84$ & & \\
\hline \multicolumn{5}{|c|}{ Remission overall ( $n=135$ attacks in 33 patients) } \\
\hline Complete & $13 / 51(25)$ & $10 / 84(12)$ & \multirow[t]{3}{*}{$0.06^{\mathrm{d}}$} & \multirow[t]{3}{*}{$0.371(0.134-1.02)$} \\
\hline Incomplete/no & $37 / 51(73)$ & $73 / 84(87)$ & & \\
\hline MD & $1 / 51(2)$ & $1 / 84(1)$ & & \\
\hline \multicolumn{5}{|c|}{ Remission HD-S (first pulse) ( $n=114$ attacks in 32 patients) } \\
\hline Complete & $11 / 45(24)$ & $9 / 69(13)$ & \multirow[t]{3}{*}{$0.12^{\mathrm{d}}$} & \multirow[t]{3}{*}{$0.417(0.139-1.255)$} \\
\hline Incomplete/no & $33 / 45(73)$ & $59 / 69(86)$ & & \\
\hline MD & $1 / 45(2)$ & $1 / 69(1)$ & & \\
\hline \multicolumn{5}{|c|}{ Remission TPE (first cycle) ( $n=$ eight attacks in five patients) } \\
\hline Complete & $0 / 0$ & $1 / 8(13)$ & \multirow[t]{2}{*}{ NA } & \\
\hline Incomplete/no & $0 / 0$ & $7 / 8(87)$ & & \\
\hline \multicolumn{5}{|c|}{ Remission immunoadsorption (first cycle) ( $n=$ one attack in one patient) } \\
\hline Complete & $1 / 1(100)$ & $0 / 0(0)$ & \multirow[t]{2}{*}{ NA } & \\
\hline Incomplete/no & $0 / 1(0)$ & $0 / 0(0)$ & & \\
\hline \multicolumn{5}{|c|}{$\begin{array}{l}\text { HD-S: high-dose intravenous steroids; TPE: therapeutic plasma exchange; NA: not applicable; MD: missing data; CI: confidence } \\
\text { interval; SD: standard deviation; NMO: neuromyelitis optica; NMOSD: neuromyelitis optica spectrum disorder; AQP4-ab: aquapo- } \\
\text { rin-4 antibody; GEE: generalized estimating equation. } \\
\text { Shown are median and interquartile range (IQR) or mean } \pm \text { SD when normally distributed. } \\
\text { aExact Mann-Whitney test. } \\
\text { bExact chi-square test. } \\
\text { 'NMO fulfilling Wingerchuk criteria. }{ }^{10} \\
\text { dGEE analysis. }\end{array}$} \\
\hline
\end{tabular}

availability of AQP4-ab testing, up to $42 \%$ of NMO patients were misdiagnosed as MS. ${ }^{4}$ The higher prevalence of MS compared to NMO and the fact that NMO was considered to be a form of MS might be the reasons for this finding. We did not observe differences in ARR between male and female patients; therefore, frequency of attacks does not serve as explanation for differences in duration between onset and diagnosis. Given the severity of attacks in NMO/SD and the differences in treatment response between $\mathrm{NMO} / \mathrm{SD}$ and $\mathrm{MS},{ }^{20,21}$ an early correct diagnosis is essential. ${ }^{22}$

Besides prevalence, clinical features in autoimmune diseases are known to be influenced by sex. In our cohort, AQP4-abs were more frequent in female patients. Other studies confirmed a female predominance among seropositive patients. ${ }^{4,5}$ Sex differences in antibody serum status are also known in other autoimmune diseases. Of note, anti-SSA and anti-SSB were detected more often in women with SLE, ${ }^{23}$ whereas anti-dsDNA were shown to be more prevalent among male patients. ${ }^{24}$ Thus, a female predominance is not necessarily found in antibody-mediated autoimmune diseases. The reasons for sex differences in the prevalence of autoreactive autoantibodies are not fully understood, but they might be of importance as $\mathrm{NMO} / \mathrm{SD}$ possibly takes a more severe course in seropositive patients. ${ }^{4,25,26}$ 
Sex differences in predisposition and course of autoimmune diseases are, first and foremost, assumed to be based on differences in immunocompetence and immune reactivity. Hormonal factors, for example, differences in circulating sex hormones or changes in hormone receptor expression are supposed to be involved in sex differences in autoimmune diseases. ${ }^{27}$ Moreover, differences in major histocompatibility complex (MHC) risk alleles, in genetic imprinting, in the transcription of inflammation-related genes, and in the responsiveness to environmental factors, for example, infections, smoking, or sun exposure, have to be taken into consideration. ${ }^{28,29}$ Women are known to show significantly stronger immune responses to infections and vaccination. ${ }^{30}$ Immunological tolerance might be more strongly controlled by hormonal factors, especially before menopause and during pregnancy.

We chose the cutoff of 40 years to compare female patients during and beyond fertile age. In general, menopause starts at an age of 50 years and is characterized by the last menstrual period. However, a decline in fertility already starts up to 10 years before, as pregnancy rates are continuously decreasing after the age of 40 years. ${ }^{12}$ The decline in reproductive function of the ovaries is accompanied by various hormonal changes, for example, of growth hormone, follicle-stimulating hormone, and estradiol, ${ }^{31}$ which might have an influence on autoimmunity. ${ }^{32,33}$

In women, response to attack treatment was age related. Complete remission and a better response to treatment with HD-S were found more frequently at an age $<40$ years. We did not observe the same age dependency in male patients, which might primarily be due to the small sample size of the male group. In other diseases, for example, in MS, age seems to influence attack severity and recovery. ${ }^{34}$ Some studies described an overall better recovery from MS attacks in young-onset patients, ${ }^{18}$ whereas others found no differences or even reverse results. ${ }^{35}$ However, in these studies, the influence of different treatment regimens was not explicitly considered.

The retrospective design of our study is a potential limitation. However, NMO/SD is a very rare disorder and was recognized as a disease entity distinct from MS only a few years ago. Thus, to date, only retrospective analyses allow to investigate epidemiologic and clinical features in large cohorts of NMO/SD patients. Another limitation is the small sample size of the male and pediatric subgroups in our cohort. Although the NEMOS cohort is one of the largest $\mathrm{NMO} / \mathrm{SD}$ cohorts published to date, our study includes only 34 male patients and six children $<15$ years. The cutoffs for fertile age used in this study are necessarily arbitrary (15-40years), as the beginning of menstrual irregularities was not systematically recorded in our study. Desirable for further studies would be the use of time-dependent covariates (exact date of last and first menses) in the statistical analysis. Finally, no structured pregnancy data were recorded in our study, which is another limitation.

As a major strength and different from previous studies, our data are derived from a very large cohort from a country where almost everybody has access to the healthcare system, reducing a bias toward more severely affected patients as well as potential genetic confounders.

Given the results from this retrospective, exploratory study, larger prospective studies are now warranted. A novelty of our study was to include (although arbitrary) cutoffs for reproductive age; this should also be considered in future studies. Such future studies should investigate potential confounders related to long-term treatment in more detail, should include more male patients and the exact dates of menarche and menopause to determine the real "fertile age." Our study provides a strong rationale for future prospective studies exploring the effect of sex as well for the inclusion of respective subgroup analyses in future treatment trials in $\mathrm{NMO} / \mathrm{SD}$.

\section{Acknowledgements}

We would like to thank all patients for participating in the study. We thank Gerda Siebert, CRO SOSTANA GmbH Berlin, Berlin, Germany, for performing statistical analysis. Members of the Neuromyelitis Optica Study Group (NEMOS) are listed below in alphabetical order. All institutions are in Germany, unless otherwise indicated. P. Albrecht, University of Düsseldorf; O. Aktas, University of Düsseldorf; K. Angstwurm, University of Regensburg; I. Ayzenberg, Ruhr University Bochum; A. Berthele, Technical University Munich; F. Bischof, University of Tübingen; N. Borisow, Charité University Medicine Berlin; T. Böttcher, Bonhoeffer Klinikum Neubrandenburg; J. Brettschneider, University of Ulm; M. Buttmann, University of Würzburg; B. Ettrich, University of Leipzig; J. Faiss, Asklepios Klinik Teupitz; A. Gass, University Hospital Mannheim; C. Geis, University of Jena; K. Guthke, Klinikum Görlitz; J. Havla, Ludwig Maximilians University Munich; H.-P. Hartung, University of Düsseldorf; K. Hellwig, Ruhr University Bochum; B. Hemmer, Technical University Munich; F. Hoffmann, Krankenhaus Martha-Maria Halle; U. Hofstadt-van 
Oy, Klinikum Westfalen Dortmund; M. Hümmert, Hannover Medical School; S. Jarius, University of Heidelberg; M. Kaste, Nordwest-Krankenhaus Sanderbusch; P. Kermer, Nordwest-Krankenhaus Sanderbusch; P. Kern, Asklepios Klinik Teupitz; C. Kleinschnitz, University Hospital Essen; I. Kleiter, RuhrUniversity Bochum;W.Köhler,Fachkrankenhaus Hubertusburg; E. Kolesilova, Asklepios Klinik Teupitz; M. Krumbholz, Ludwig Maximilians University Munich; T. Kümpfel, Ludwig Maximilians University Munich; S. Langel, Landeskrankenhaus Rheinhessen; F. Lauda, University of Ulm; M. Liebetrau, Evangelische Bathildiskrankenhaus Bad Pyrmont gGmbH; R. Linker, University of Erlangen; W. Marouf, Heliosklinik Stralsund; M. Marziniak, Isar-Amper Klinik Ost Munich; A. Melms, University of Erlangen; I. Metz, University of Göttingen; C. Mayer, University of Frankfurt; C. Münch, Charité University Medicine Berlin; O. Neuhaus, SRH Krankenhaus Sigmaringen; S. Niehaus, Klinikum Dortmund; F. Pache, Charité University Medicine Berlin; F. Paul, Charité University Medicine Berlin, H. Pellkofer, University of Göttingen; A. Riedlinger, Asklepios Klinik Teupitz; M. Ringelstein, University of Düsseldorf; L. Röpke, University of Jena; S.P. Rommer, University of Vienna (Austria); K. Ruprecht, Charité University Medicine Berlin; C. Ruschil, University of Tübingen; S. Schippling, University of Zürich (Switzerland); S. Schuster, University of Hamburg; M. Schwab, University of Jena; M. Stangel, Hannover Medical School, J. Stellmann, University of Hamburg; M. Stoppe, University of Leipzig; F. Then Bergh, University of Leipzig; C. Trebst, Hannover Medical School; J. Tünnerhoff, University of Tübingen; H. Tumani, University of Ulm; C. Veauthier, Charité University Medicine Berlin; A. Walter, Klinikum Herford; K.P. Wandinger, Institute of Clinical Chemistry, Neuroimmunology Unit, and Department of Neurology, University Medical Center Schleswig-Holstein Campus Lübeck; M.S. Weber, University of Göttingen; R. Weissert, University of Regensburg; B. Wildemann, University of Heidelberg; C. Wilke, Nervenzentrum Potsdam; A. Winkelmann, University of Rostock; K. Young, University of Hamburg; L. Zeltner, University of Tübingen; C. Zentner, Martha-Maria, University of Halle; U. Zettl, University of Rostock; U. Ziemann, University of Tübingen.

\section{Declaration of Conflicting Interests}

The author(s) declared the following potential conflicts of interest with respect to the research, authorship, and/or publication of this article: P.A. has received honoraria for speaking/consultation and travel grants from Biogen Idec, Novartis, Teva, Merz
Pharmaceuticals, and Ipsen and research grants from Biogen Idec, Teva and Novartis Pharmaceuticals. O.A. has received honoraria for speaking/consultation and travel grants from Bayer Healthcare, Biogen Idec, Chugai, Novartis, Merck Serono, and Teva and research grants from Bayer Healthcare, Biogen Idec, Novartis, and Teva. A.B. has received a research grant on NMO from Bayer Healthcare. He has received honoraria for speaking/consultation and travel grants from Bayer Healthcare, Biogen Idec, Merck Serono, Genzyme, Novartis, and Teva. N.B. has received a research grant from Alexion Pharmaceuticals, Inc. M.B. received honoraria for speaking/consultation and travel grants from Bayer, Biogen, Genzyme, Novartis, Roche, and Teva. He received research support from Merck Serono and Novartis. C.G. has received honoraria for speaking/consultation and travel grants from Teva Pharma GmbH, Merck Serono, Biogen Idec, Novartis Pharmaceuticals, CSL Behring, and Allergan and research grants from Merck Serono, Novartis Pharmaceuticals, and CSL Behring. H.P.H. received honoraria for consultancy and speaking from Bayer Health Care, Biogen Idec, GeNeuro, Genzyme, Novartis, Opexa, Teva, Sanofi-Aventis, and Roche and holds patents with permission by the Rector of Heinrich-Heine-University Düsseldorf. J.H. received speaker honoraria, travel expenses, and personal compensations from Merck Serono, Biogen, Bayer Healthcare, and Novartis Pharma. K.H. received research grants and speaker honoraria from Bayer Healthcare, Biogen Idec Germany, Merck Serono, Novartis Pharma and Teva Pharma and Sanofi-Aventis Genzyme Pharmaceuticals. B.H. has served on scientific advisory boards for Roche, Novartis, Bayer Schering, Merck Serono, Biogen Idec, GSK, Chugai Pharmaceuticals, Micromet, and Genzyme Corporation; has received speaker honoraria from Bayer Schering, Novartis, Biogen Idec, Merck Serono, Roche, and Teva Pharmaceutical Industries Ltd; and has received research support from Biogen Idec, Bayer Schering, Merck Serono, Five prime, Metanomics, Chugai Pharmaceuticals, and Novartis. He has filed a patent for the detection of antibodies and $\mathrm{T}$ cells against KIR4.1 in a subpopulation of MS patients. F.H. received honoraria for consultancy and speaking and travel grants from Allergan, Bayer, Biogen, Boehringer Ingelheim, CSL Behring, Diamed Medizintechnik, Genzyme, Grifols, Ipsen, Merck Serono, Merz, Novartis, Octapharm, Pfizer, Teva, Talecris, UCB. U.H. has received honoraria for speaking/consultation and travel grants from Bayer Healthcare, Biogen Idec, Merck Serono, Genzyme, a Sanofi Company, MEDA Pharma, Novartis Pharmaceuticals, and Teva Pharma GmbH and research grants from Bayer Healthcare and Merck 
Serono. S.J. was indirectly supported by research grants from Merck Serono, and Bayer Healthcare to the Department of Neurology at the University of Heidelberg. P.K. has received honoraria for speaking/ consulting and research/travel grants from Abbvie, Bayer Healthcare, Biogen, Boehringer, Bristol-Myers Squibb, Genzyme, MSD, Novartis, Pfizer, TEVA, and UCB. I.K. has received honoraria for speaking/consultation and travel grants from Bayer Healthcare, Biogen Idec, and Chugai and research grants from Bayer Healthcare, Biogen Idec, Chugai, Novartis Pharmaceuticals, and Diamed. M.K. received grant support and traveling expenses from Novartis Pharmaceuticals. T.K. has received travel expenses and speaking honoraria from Bayer Healthcare, Genzyme, Teva Pharma, Merck Serono, Novartis, Sanofi-Aventis, and Biogen Idec as well as grant support from Bayer Schering AG, and Novartis. F.L. received funding for travel from Teva Pharma. R.L. has received honoraria for speaking/consultation and travel grants from Bayer Healthcare, Biogen Idec, Genzyme GmBH, Merck Serono, Novartis Pharmaceuticals, and TEVA Pharma $\mathrm{GmbH}$ and research support from Biogen Idec, Merck Serono, and Novartis Pharmaceuticals. M.M. has received honoraria for speaking/consultation and travel grants from Bayer Healthcare, Biogen Idec, Merck Serono, Genzyme, a Sanofi Company, Novartis Pharmaceuticals, and Teva Pharma $\mathrm{GmbH}$ and research grants from Biogen Idec and Novartis Pharmaceuticals. C.M. has received honoraria for speaking/consultation and travel grants from Bayer Healthcare, Biogen Idec, Merck Serono, Genzyme, a Sanofi Company, Novartis Pharmaceuticals, and Teva Pharma GmbH and research grants from Novartis Pharmaceuticals. F.Pac. has received funding from a research grant from Novartis Pharmaceuticals and travel grants from Genzyme. The position of F.Pac. was supported by a grant of the Bundesministerium für Bildung und Forschung (Competence Network Multiple Sclerosis) to F.Pau. F.Pac. is participant in the BIH-Charite Clinical Scientist Program funded by the CharitéUniversitätsmedizin Berlin and the Berlin Institute of Health. F.Pau. has received honoraria for speaking/ consultation and travel grants from Alexion, Bayer Healthcare, Biogen Idec, Novartis, MedImmune, Merck Serono, Genzyme, and Teva and research grants from the German Research Foundation, the German Ministry of Education and Research (BMBF/ KKNMS, Competence Network Multiple Sclerosis). M.R. received speaker honoraria from Novartis and travel reimbursement from Bayer Schering, Biogen Idec, and Genzyme with permission by the Rector of Düsseldorf University Hospital. P.R. has received travel grants, consultancy, and speaking honoraria from Novartis and Biogen as well as honoraria for scientific lectures from Genzyme. He has received travel grants from Teva. K.R. has received research support from Novartis as well as speaking fees and travel grants from Bayer Healthcare, Biogen Idec, Merck Serono, Sanofi-Aventis/Genzyme, Teva Pharmaceuticals, and Novartis. M.Sc. has received research support from Bayer Healthcare and Novartis as well as honoria for speaking fees/consultations and travel grants from Bayer Healthcare, Biogen Idec, Merck Serono, Genzyme, Teva Pharmaceuticals, and Novartis. M.St. has received honoraria for scientific lectures or consultancy from Bayer Healthcare, Biogen Idec, Baxter, CSL Behring, Grifols, Merck Serono, Novartis, Sanofi-Aventis, and Teva. His institution received research support from Bayer Healthcare, Biogen Idec, Merck Serono, Novartis, and Teva. J.P.S. has received honoraria for scientific lectures from Bayer Healthcare, Biogen, Novartis, and Genzyme. His institution received research support from Bayer Healthcare, Biogen, Merck Serono, and Novartis. Mu.S. has received research support for investigatorinitiated studies, has served on advisory boards, and received travel support to scientific meetings from Biogen Idec, Fresenius, Merck Serono, Novartis, and Teva. F.T.B. has received honoraria for speaking, has served on advisory boards, and received travel grants from Bayer Healthcare, Biogen Idec, CSL Behring, Genzyme, Merck Serono, Novartis and Teva. He has received research grants from Bayer Healthcare, Fresenius, Novartis Pharma GmbH, and Teva Pharma $\mathrm{GmbH}$. C.T. has received honoraria for speaking, consultation, and expert testimony and participation in advisory boards from Bayer Vital GmbH, Biogen Idec, Genzyme GmbH, Novartis Pharmaceuticals, and Sanofi-Aventis Deutschland GmbH. H.T. has received honoraria for speaking/consultation and travel grants from Bayer Healthcare, Biogen Idec, Merck Serono, Genzyme, Novartis Pharma, Siemens Health Products, and Teva Pharma and research grants from Biogen Idec, Merck Serono, Novartis Pharma, Siemens Health Products, and Teva Pharma. C.V. has received travel grants from Genzyme and Teva Pharma. A.W. has received honoraria for speaking/consultation and travel grants from Bayer HC, Biogen, Diamed, Merck Serono, Genzyme, Sanofi, Teva, Novartis, and Roche. R.W. received consulting fees from Biogen, Genzyme, Merck Serono, Novartis, Roche, and Teva and performed contracted research for Novartis. B.W. has received honoraria for speaking/consultation and travel grants from Bayer Healthcare, Biogen Idec, Merck Serono, Genzyme, a Sanofi Company, Novartis Pharmaceuticals, and Teva Pharma GmbH and research grants from Biogen Idec, Biotest, Merck Serono, Novartis Pharmaceuticals, and Teva Pharma GmbH. A.W. has received speaker's honoraria and 
travel expense compensation from Bayer Health Care, Novartis, Biogen, Genzyme, and Merck Serono. U.Z. has received honoraria from Biogen Idec, Deutschland $\mathrm{GmbH}$, Bayer Vital GmbH, Bristol-Myers Squibb $\mathrm{GmbH}$, CorTec $\mathrm{GmbH}$, Medtronic, and Servier for advisory work and grants from Biogen Idec and Janssen Pharmaceuticals NV for supporting investigator-initiated trials. A. Gah. received travel reimbursement from Sanofi Genzyme. K.A, I.A., F.B., T.B., J.B., B.E., J.F., K.F., A.Gas., K.G., M.K., P.K., C.K., W.K., E.K., S.L., W.M., A.M., I.M., C.M., O.N., S.N., H.P., A.R., L.R., C.R., S.Sve., S.Sim., J.T., K-P.W., M.W., B.W., K-D.W., C.W., K.Y., L.Z., C.Z., and U.Z. declare no conflict of interest.

\section{Funding}

The author(s) disclosed receipt of the following financial support for the research, authorship, and/or publication of this article: The NEMOS cohort/NationNMO is supported by the German Ministry for Education and Research (BMBF) as part of the German Competence Network Multiple Sclerosis (KKNMS; for NEMOS NationNMO-DAB FKZ 01GI1602C to J.S. and NationNMO-PAT FKZ 01GI1602B to O.A.).

\section{References}

1. Jarius S, Wildemann B and Paul F. Neuromyelitis optica: Clinical features, immunopathogenesis and treatment. Clin Exp Immunol 2014; 176: 149-164.

2. Wingerchuk DM. Neuromyelitis optica: Effect of gender. J Neurol Sci 2009; 286: 18-23.

3. Mealy MA, Wingerchuk DM, Greenberg BM, et al. Epidemiology of neuromyelitis optica in the United States: A multicenter analysis. Arch Neurol 2012; 69: $1176-1180$.

4. Jarius S, Ruprecht K, Wildemann B, et al. Contrasting disease patterns in seropositive and seronegative neuromyelitis optica: A multicentre study of 175 patients. J Neuroinflammation 2012; 9: 14.

5. Siritho S, Apiwattanakul M, Nakashima I, et al. Features of anti-aquaporin 4 antibody-seronegative Thai patients with neuromyelitis optica spectrum disorders: A comparison with seropositive cases. $J$ Neurol Sci 2014; 341: 17-21.

6. Wingerchuk DM and Weinshenker BG. Neuromyelitis optica clinical predictors of a relapsing course and survival. Neurology 2003; 60: 848-853.

7. Lisnevskaia L, Murphy G and Isenberg D. Systemic lupus erythematosus. Lancet 2014; 384: 1878-1888.

8. Bove R and Chitnis T. Sexual disparities in the incidence and course of MS. Clin Immunol Orlando Fla 2013; 149: 201-210.
9. Schwendimann RN and Alekseeva N. Gender issues in multiple sclerosis. Int Rev Neurobiol 2007; 79: 377-392.

10. Wingerchuk DM, Lennon VA, Pittock SJ, et al. Revised diagnostic criteria for neuromyelitis optica. Neurology 2006; 66: 1485-1489.

11. Wingerchuk DM, Lennon VA, Lucchinetti CF, et al. The spectrum of neuromyelitis optica. Lancet Neurol 2007; 6: 805-815.

12. Poetzsch O, Weinmann J and Haustein T. Birth trends and the family situation in Germany. Report, Federal Statistical Office, Wiesbaden, Germany, 2012.

13. Stepaniak U, Szafraniec K, Kubinova R, et al. Age at natural menopause in three central and eastern European urban populations: The HAPIEE study. Maturitas 2013; 75: 87-93.

14. Kleiter I, Gahlen A, Borisow N, et al. Neuromyelitis optica: Evaluation of 871 attacks and 1153 treatment courses. Ann Neurol 2016; 79: 206-216.

15. Dahmen $G$ and Ziegler A. Generalized estimating equations in controlled clinical trials: Hypotheses Testing. Biom J 2004; 46: 214-232.

16. Alamanos Y, Voulgari PV, Siozos C, et al. Epidemiology of systemic lupus erythematosus in northwest Greece 1982-2001. J Rheumatol 2003; 30: 731-735.

17. Huppke B, Ellenberger D, Rosewich H, et al. Clinical presentation of pediatric multiple sclerosis before puberty. Eur J Neurol 2014; 21: 441-446.

18. Cossburn M, Ingram G, Hirst C, et al. Age at onset as a determinant of presenting phenotype and initial relapse recovery in multiple sclerosis. Mult Scler Houndmills Basingstoke Engl 2012; 18: 45-54.

19. McKeon A, Lennon VA, Lotze T, et al. CNS aquaporin-4 autoimmunity in children. Neurology 2008; 71: 93-100.

20. Uzawa A, Mori M, Hayakawa S, et al. Different responses to interferon beta- $1 \mathrm{~b}$ treatment in patients with neuromyelitis optica and multiple sclerosis. Eur J Neurol Off J Eur Fed Neurol Soc 2010; 17: 672-676.

21. Kleiter I, Hellwig K, Berthele A, et al. Failure of natalizumab to prevent relapses in neuromyelitis optica. Arch Neurol 2012; 69: 239-245.

22. Trebst C, Jarius S, Berthele A, et al. Update on the diagnosis and treatment of neuromyelitis optica: Recommendations of the Neuromyelitis Optica Study Group (NEMOS). J Neurol 2014; 261: 1-16.

23. López P, Mozo L, Gutiérrez C, et al. Epidemiology of systemic lupus erythematosus in a northern Spanish population: Gender and age influence on immunological features. Lupus 2003; 12: 860-865. 
24. Borba EF, Araujo DB, Bonfá E, et al. Clinical and immunological features of 888 Brazilian systemic lupus patients from a monocentric cohort: Comparison with other populations. Lupus 2013; 22: 744-749.

25. Akman-Demir G, Tüzün E, Waters $P$, et al. Prognostic implications of aquaporin-4 antibody status in neuromyelitis optica patients. J Neurol 2011; 258: 464-470.

26. Huppke P, Blüthner M, Bauer O, et al. Neuromyelitis optica and NMO-IgG in European pediatric patients. Neurology 2010; 75: 1740-1744.

27. McCombe PA, Greer JM and Mackay IR. Sexual dimorphism in autoimmune disease. Curr Mol Med 2009; 9: 1058-1079.

28. Ponsonby A-L, Lucas RM, van der Mei IA, et al. UVR, vitamin D and three autoimmune diseasesMultiple sclerosis, type 1 diabetes, rheumatoid arthritis. Photochem Photobiol 2005; 81: 1267-1275.

29. Voskuhl RR and Gold SM. Sex-related factors in multiple sclerosis susceptibility and progression. Nat Rev Neurol 2012; 8: 255-263.
30. Furman D, Hejblum BP, Simon N, et al. Systems analysis of sex differences reveals an immunosuppressive role for testosterone in the response to influenza vaccination. Proc Natl Acad Sci U S A 2014; 111: 869-874.

31. Batrinos ML. Premenopause: The endocrinology of reproductive decline. Horm Athens Greece 2013; 12: 334-349.

32. Athreya BH, Pletcher J, Zulian F, et al. Subset-specific effects of sex hormones and pituitary gonadotropins on human lymphocyte proliferation in vitro. Clin Immunol Immunopathol 1993; 66: 201-211.

33. Kovats S. Estrogen receptors regulate innate immune cells and signaling pathways. Cell Immunol 2015; 294: 63-69.

34. Kalincik T, Buzzard K, Jokubaitis V, et al. Risk of relapse phenotype recurrence in multiple sclerosis. Mult Scler Houndmills Basingstoke Engl 2014; 20: 1511-1522.

35. West T, Wyatt M, High A, et al. Are initial demyelinating event recovery and time to second event under differential control? Neurology 2006; 67 : 809-813.
Visit SAGE journals online journals.sagepub.com/ home/msj

(SAGE journals 